\section{RHINOSCLEROMA IN A 5-YEAR-OLD PORTUGUESE CHILD}

\author{
Inês Simão, MD, * Iuri Gaspar, MD, † Rosário Faustino, MD, † \\ and Maria João Rocha Brito, $M D^{*}$
}

Abstract: Rhinoscleroma is a chronic granulomatous infectious disease that is rare in Western Europe. We report the case of a 5-year-old Portuguese boy diagnosed with rhinoscleroma in the context of recurrent epistaxis. He had a 6-month course of antibiotic (amoxicillin plus clavulanate) therapy with full recovery.

Key Words: rhinoscleroma, Klebsiella rhinoscleromatis, epistaxis, children

Accepted for publication December 23, 2013.

From the *Infectious Disease Unit, Department of Pediatrics H. Dona Estefânia, Centro Hospitalar Lisboa Central, Lisbon; and †Otorhinolaryngology Department, Hospital Distrital de Santarém, Santarém, Portugal

The authors have no funding or conflicts of interest to disclose.

Address for correspondence: Inês Simão, MD, Infectious Disease Unit - Department of Pediatrics H. Dona Estefânia, Centro Hospitalar Lisboa Central, Lisbon, Portugal. E-mail: isvs1@sapo.pt

Copyright (C) 2014 by Lippincott Williams \& Wilkins

DOI: $10.1097 /$ INF.0000000000000263

$\mathrm{R}^{\mathrm{s}}$ hinoscleroma is a chronic granulomatous infectious disease of the upper respiratory tract caused by the Gram-negative coccobacillus Klebsiella rhinoscleromatis, a subspecies of Klebsiella pneumoniae..$^{1,2}$ Despite being broadly distributed in nature, it has not been found in a nonhuman host. ${ }^{2}$ It is endemic in some regions of Africa (Egypt, tropical areas), Southeast Asia, Mexico, Central and South America and Central and Eastern Europe. Although first reported in Europe, nowadays only a few sporadic cases have been described in Western Europe, probably the result of emigration of people from endemic areas. ${ }^{1}$

Rhinoscleroma can affect most parts of the respiratory tract and is classified in 3 overlapping stages: catarrhal or atrophic (sometimes called ozaena), granulomatous (proliferative or nodular) and sclerotic (cicatricial or fibrotic). ${ }^{3}$ Most patients are diagnosed in the granulomatous stage, in which the typical histologic characteristics can be observed. ${ }^{3}$ Culture of biopsy specimens is the most useful diagnostic test to distinguish it from other granulomatous diseases with identical histologic characteristics, but culture is positive only in half the patients during the granulomatous stage. ${ }^{234}$

Treatment includes long-term antibiotics and surgery in patients with obstructive symptoms. ${ }^{14,5}$ We report the case of rhinoscleroma in a 5-year-old previously healthy Portuguese boy, who responded completely to treatment with amoxicillin-clavulanate.

\section{CASE REPORT}

A 5-year-old Portuguese boy, the second child of a non-consanguineous couple (Fig. 1A) was brought to the Emergency Department with epistaxis for the 2 previous weeks with no other accompanying signs or symptoms. The child and his family had gone on vacation to the Dominican Republic the year before. The medical histories of his 10-year-old sister and parents were uneventful. Rhinoscopy revealed an intranasal bleeding mass (Fig. 1B) and magnetic resonance imaging confirmed the intranasal mass with extension to the bone of the ethmoid sinus (Fig. 1C). Histopathology revealed chronic inflammation, granulomas and Mikulicz cells (large vacuolated histiocytes) with numerous intracellular Gramnegative bacilli (Fig. 1D). Cultures of biopsy samples in MacConkey agar were positive for $K$. rhinoscleromatis that was susceptible to amoxicillin-clavulanate. Screening tests for immunodeficiency
A
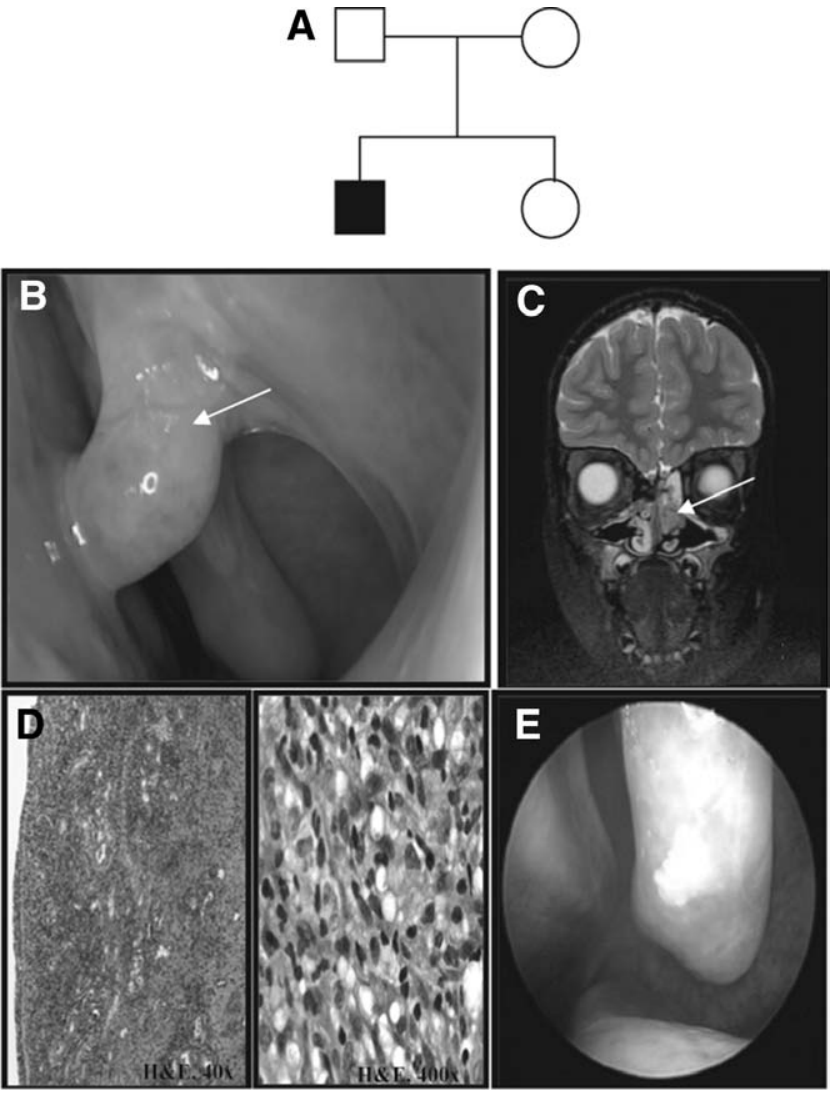

FIGURE 1. A) Pedigree of the family. Squares, male subjects; circles, female subjects; closed symbols, index case; open symbols, unaffected individuals. B) First rhinoscopy: middle turbinate and meatus mass. C) Coronal magnetic resonance imaging scan (T2-weighted): intranasal mass with extension to the ethmoid bone sinus. D) Histopathologic characteristic features (granulomas containing Mikulicz cells). E) Last rhinoscopy: middle turbinate and meatus without lesions.

(immunoglobulins, lymphocyte subpopulations, phagocytosis test, oxidative burst and $\mathrm{CH} 100$ ) were normal. Genetic studies were negative for the DQA $1 * 03011-\mathrm{DQB} 1 * 0301$ haplotype.

He was treated with amoxicillin plus clavulanate (4:1) $50 \mathrm{mg} / \mathrm{kg} / \mathrm{d}$, every 8 hours per os, for 6 months. Within a month, his symptoms resolved and the intranasal mass was significantly reduced. There was progressive reduction and eventual total disappearance of macro and microscopic lesions when therapy had been given for 6 months. After 3 years of follow up, he remains asymptomatic and rhinoscopy was normal (Fig. 1E).

\section{DISCUSSION}

$K$. rhinoscleromatis is transmitted by direct inhalation of droplets. ${ }^{3}$ Person-to-person transmission probably occurs by airborne secretions, but other risk factors such as overcrowded living facilities, poor hygiene and poor nutrition may be necessary for the disease to develop. ${ }^{2,5}$ In the current case, none of these conditions was verified. The only relevant history was a trip to the Dominican Republic, an endemic region.

A Mexican study showed that DQA $1 * 03011-\mathrm{DQB} 1 * 0301$ haplotype is also a risk factor for developing Rhinoscleroma. ${ }^{6}$ Our patient's genetic study was negative. Impaired cellular immunity 
with low CD4+ lymphocyte count and high CD8+ lymphocyte count and with decreased CD4/CD8 ratio within the lesion, possibly inducing a diminished or altered T-cell response, may be responsible for an increased host susceptibility to the development of rhinoscleroma. ${ }^{5}$ Humoral immune response itself does not control the infection, due to not only the bacterial intracellular nature and thick mucin coat but also the nonspecific nature of the host's antibody response..$^{5}$ In the present case, screening tests for immunodeficiency were normal.

Rhinoscleroma can affect most parts of the respiratory tract. ${ }^{7}$ Infection usually starts in the subepithelium of the nasal mucosa, with permanent damage in most cases, spreading thereafter to other areas. $^{7}$ Eventually, subepithelial invasion by neutrophils and histiocytes can extend to the interstitium as well as to the adjacent cartilage and bone. ${ }^{7}$ In our patient, the mass was limited to the intranasal cavity and the bone lining of the ethmoid sinus.

Rhinoscleroma is classified in 3 overlapping stages described earlier. ${ }^{3}$ As observed in this case, most patients are diagnosed in the granulomatous stage, because this is the most symptomatic phase and exhibits characteristic histologic features (Mikulicz cell-large phagocytes with a single shrunken nucleus, displaced to the cell periphery because of the presence of multibacillary cytoplasmatic vacuoles; transformed plasma cells with Russell bodies; chronic inflammatory cells and pseudoepitheliomatous hyperplasia). ${ }^{8}$ Our patient, as described in literature, culture of biopsy specimens in MacConkey agar confirmed the diagnosis. ${ }^{3}$

Treatment includes surgery in patients with obstructive symptoms and long-term antimicrobial therapy in an attempt to eradicate $K$. rhinoscleromatis, because the relapse rate is high. ${ }^{1,3}$ In vitro, these bacteria are inhibited by clinically achievable concentrations of amoxicillin-clavulanate, chloramphenicol, trimethoprim-sulfamethoxazole, cephalosporins, streptomycin, tetracyclines and ciprofloxacin. ${ }^{3}$ However, in vivo, effective antibiotics are streptomycin, doxycycline, tetracycline, rifampicin, sulfonamides, clofazimine, ciprofloxacin and ofloxacin... ${ }^{2,3}$ Because of our patient's age, antibiotic choice was challenging, as most of the recommended drugs present risk of serious side effects in this age group. We decided to initiate a prolonged course (6 months) of amoxicillin plus clavulanate (4:1). The choice was based on the following criteria: broad spectrum of bactericidal activity against many Gram-positive and Gram-negative microorganisms (including Klebsiella species); favorable side effect profile; availability of a pediatric formulation and good compliance in the long term. ${ }^{9}$ At present, after 3 years of follow up off antibiotherapy, the child remains asymptomatic and rhinoscleroma has clinically resolved. Several studies report relapses after initial clinical response. ${ }^{10}$ For this reason, our patient remains under annual follow up.

\section{REFERENCES}

1. Chan TV, Spiegel JH. Klebsiella rhinoscleromatis of the membranous nasal septum. J Laryngol Otol. 2007;121:998-1002.

2. de Pontual L, Ovetchkine P, Rodriguez D, et al. Rhinoscleroma: a French National Retrospective Study of Epidemiological and Clinical Features. Clin Infect Dis. 2008; 47:1396-1402.

3. Hart CA, Rao SK. Rhinoscleroma. J Med Microbiol. 2000;49:395-396.

4. Bolze A, Abhyankar A, Grant AV, et al. A mild form of SLC29A3 disorder: a frameshift deletion leads to the paradoxical translation of an otherwise noncoding mRNA splice variant. PLoS One. 2012;7:e29708

5. Maguiña C, Cortez-Escalante J, Osores-Plenge F, et al. Rhinoscleroma: eight Peruvian cases. Rev Inst Med Trop Sao Paulo. 2006;48:295-299.

6. Sánchez-Marín LA, Bross-Soriano D, Arrieta J. Association of HLA-DQA1*03011-DQB1*0301 haplotype with the development of respiratory scleroma. Otolaryngol Head Neck Surg. 2007;136:481-483

7. Canalis RF, Zamboni L. An interpretation of the structural changes responsible for the chronicity of rhinoscleroma. Laryngoscope. 2001;111:1020-1026.
8. Sood N, Sood S, Arora S, et al. Cytohistological features of rhinoscleroma. Indian J Pathol Microbiol. 2011;54:806-808.

9. Amoxicillin and Clavulanate Official FDA information, side effects and uses. [FDA drugs web site]. December, 2012. Available at: www.drugs.com/ pro/amoxicillin-and-clavulanate.html. Accessed March 1, 2013.

10. Badia L, Lund VJ. A case of rhinoscleroma treated with ciprofloxacin. J Laryngol Otol. 2001;115:220-222.

\section{SECONDARY BACTEREMIA IN ROTAVIRUS GASTROENTERITIS}

\author{
Salih Gözmen, MD, * Keskin Şükran Gözmen, $M D$, * \\ Hurşit Apa, MD, * Hüseyin Aktürk, MD, † Yelda Sorguç, MD, † \\ Nuri Bayram, MD, † Gamze Gülfidan, MD, + Yeliz Oruç, BSN, $\}$ \\ Nevbahar Yaşar, BSN, § and İlker Devrim, MD†
}

\begin{abstract}
The aim of the study was to determine the frequency of secondary bacteremia in children with rotavirus gastroenteritis and persistence or reemergence of fever. We identified 376 children with a mean of age of $14.2 \pm 10.1$ months. A significant pathogen was isolated from blood cultures in 5 patients [Enterococcus faecium $(\mathrm{n}=1)$, Klebsiella spp $(\mathrm{n}=1)$, Staphylococcus aureus $(\mathrm{n}=1)$, Raoultella planticola $(\mathrm{n}=1)$, Candida albicans $(\mathrm{n}=1) ; 1.3 \%$. The frequency of secondary bacteremia in children with rotavirus gastroenteritis is low, but it should be considered when there is fever lasting $>48$ hours or reemergence of fever.
\end{abstract}

Key Words: children, gastroenteritis, rotavirus, secondary bacteremia

Accepted for publication January 7, 2014

From the *Department of Pediatrics; †Department of Pediatric Infectious Diseases; \$Department of Microbiology and Clinical Microbiology; and $\S N u r s e$, Infection Control Committee, Dr. Behçet Uz Children's Training and Research Hospital, İzmir, Turkey.

The authors have no funding or conflicts of interest to disclose.

Address for correspondence: Salih Gözmen, MD, Dr. Behçet Uz Children's Training and Research Hospital, İzmir, Turkey. E-mail: drsalihgozmen@ gmail.com.

Copyright (C) 2014 by Lippincott Williams \& Wilkins

DOI: $10.1097 /$ INF.0000000000000324

A cute diarrheal diseases cause $25-30 \%$ of all deaths among children $<5$ years in developing countries. ${ }^{1}$ Rotavirus is one of the most important causative agents of acute gastroenteritis in children between 4 and 24 months of life. Rotavirus gastroenteritis has an annual incidence of 130 million cases and 440,000 deaths and is responsible for approximately $20 \%$ of diarrhea-associated deaths in children $<5$ years of age. ${ }^{2,3}$ Although the pathogenesis of rotavirus infection is not fully understood, the main mechanisms are suggested to be infiltration of mature enterocytes in the mid and upper part of the villi of the small intestine and alteration of the function of the small intestinal epithelium, resulting in malabsorptive diarrhea secondary to enterocyte destruction. ${ }^{4}$

The disease caused by rotavirus is usually self-limited in otherwise healthy infants. In addition to the noninfectious complications, secondary bacteremia complicating rotavirus gastroenteritis has been reported in case reports. The aim of this study was to determine the frequency of secondary bacteremia in children with rotavirus gastroenteritis and persistence or reemergence of fever.

\section{MATERIALS AND METHODS}

This is a retrospective and descriptive study. Five-hundred and forty-two subjects with rotavirus gastroenteritis admitted to Behçet Uz Children's Hospital between 2008 and 2012 were enrolled in this study. Demographic data and results of blood cultures were collected from the charts of the patients. Axillary 